\title{
«ESTUDIO PSICOMETRICO DEL TEST DE MATRICES PROGRESIVAS DE RAVEN A COLORES EN ESTUDIANTES DE PRIMARIA DE LIMA METROPOLITANA»
}

\author{
Ana Delgado Vásquez \\ Magíster en Psicología Educativa
}

\begin{abstract}
RESUMEN
Se realizó el estudio psicométrico del Test de Matrices Progresivas de Raven a colores, que es un instrumento no verbal que evalúa la habilidad para hacer comparaciones, razonar por analogía y organizar percepciones espaciales dentro de un todo relacionado sistemáticamente, se diseñó principalmente como una medida de factor "g" de Spearman o inteligencia general, que está constituida por dos componentes identificados como habilidad educativa y habilidad reproductiva.

Participaron en el estudio 2496 alumnos de las siete USEs de Lima Metropolitana que se encontraban cursando del 1ro al 6to grado de primaria, con edades entre los 6 y 11 años, seleccionados en base a un muestreo probabilístico bietápico.

Los resultados del análisis psicométrico del test demuestran que el instrumento permite obtener puntajes confiables, a través del método de consistencia interna. Asimismo, el análisis de factores permitió obtener la validez de constructo de la prueba, observándose que los ítems conforman un sólo factor que explica el $76 \%$ de la varianza de las puntuaciones. Se elaboraron los baremos correspondientes a cada grupo de edad.

El análisis de los resultados destaca la comparación con otros estudios, las diferencias entres los alumnos de los colegios estatales y los no estatales, y la diferencia encontrada entre alumnas y alumnos. Se utilizan criterios educacionales y sociales en la discusión y explicación de los resultados.
\end{abstract}

Palabras clave: Matrices Progresivas de Raven a colores, inteligencia general, validez, confiabilidad, baremos, factor " $\mathrm{g}$ ".

\begin{abstract}
It was made a psychometric study of Raven Coloured Progressive Matrices Test, which is a non verbal instrument that evaluates abilities related to make comparisons, thinking for analogy and spatial perceptions organization. It was designed mainly as a measure of factor "g" of Spearman or general intelligence, composed by two abilities, named educational and reproductive.

The participants were 2496 students obtained at random considering all school districts (USE) of the city of Lima, Peru. They were primary level students, from $1^{\circ}$ to $6^{\circ}$ grade and 6 to 11 years old.

The psychometric analysis shows that the item-test relation is up to norms $(>0.20)$, so reliability was attained. Also the factor analysis of the items comes up whith one factor explainig the $76 \%$ of variance, so construct validity was obtained. Also the calification norms was obtained.

The analysis of results points out the comparison with other studies, the difference among students from public and private schools, and the difference that female and male students showed. Educational and social variables are used at formulating an explanation.
\end{abstract}

Keywords: Raven's Progressive Matrices colors, general intelligence, validity, reliability, scales, factor "g".

Miembros: Luis Escurra Mayaute,. María Rosario Quesada Murillo,. Jaime Aliaga Tovar, Carmen Alvarez Taco, María Atalaya Pisco, Mario Bulnes Bedón. Rosa Huerta Rosales, Juan Pequeña Constantino. Colaboradores: Willian Torres Acuña. 


\section{INTRODUCCIÓN}

Las Matrices Progresivas fueron construidas como medida del factor de inteligencia general de Spearman (Aiken, 1996; Anastasi y Urbina 1998). Esta prueba permite medir la capacidad de una persona para comprender figuras sin significado viendo las relaciones que hay entre ellas, captar la estructura de estas figuras completando cada sistema de relaciones y haciendo esto, desarrollar un método sistemático de razonamiento. Esto implica la aptitud para dar sentido a un material desorganizado o confuso, para manejar constructos claramente no verbales que facilitan la captación de una estructura compleja. Se trata de ir más allá de la percepción simple de algo que es obvio; es un tipo de intuición o percepción de golpe de la "solución del problema" (Raven, Court y Raven,1996).

Spearman en 1927 (Raven, Court y Raven, 1996; Anastasi y Urbina, 1998) había observado ya que la naturaleza, origen y efectos de esta capacidad era diferente de la que exige la "reproducción" de material cultural de tipo verbal (dominio, recuerdo y expresión), ese factor "cristalizado" o cultural. Ambos aspectos de la inteligencia están relacionados, porque la percepción y el pensamiento dependen, generalmente, de constructos adquiridos, y la capacidad de asimilar información depende a menudo de la habilidad para encontrar significado en un conjunto confuso de datos.

De acuerdo con la teoría bifactorial, el propósito de la evaluación psicológica debería ser la medición de la cantidad de "g" que posee cada individuo, ya que al encontrarse en todas las habilidades, es la única base para predecir la ejecución de una persona en distintas ocasiones. Sería inútil medir los factores específicos, ya que, por definición, cada uno opera en una única actividad. Por esta razón, Spearman propuso sustituir la heterogénea colección de reactivos de los tests de inteligencia con una sola prueba que tuviera una elevada saturación de "g". Además, sugería utilizar los instrumentos que evalúan las relaciones abstractas por considerar que son los que pueden hacer una mejor medición de "g". Entre los instrumentos construidos como medidas del factor "g" se encuentran las Matrices Progresivas a Colores de Raven.

Raven et al. (1991, 1996) distinguieron 5 niveles cualitativos de desarrollo en la capacidad intelectual:

a. Los niños primeros son capaces de distinguir figuras idénticas de figuras diferentes, y más tarde figuras similares de las disímiles.

b. Algún tiempo después de esto, ellos son capaces de apreciar la orientación de una figura con respecto a sí mismos y con respecto a otros objetos en el campo perceptual.

c. Después, ellos pueden comparar cambios análogos en las características percibidas y ,adoptar éste como un método lógico de razonamiento.

d. Subsiguientemente, ellos son capaces de analizar el todo percibido en sus elementos constitutivos o "caracteres", y son capaces de distinguir entre lo que se les da de aquello con lo que ellos contribuyen.

e. Finalmente, ellos son capaces de captar dos o más figuras discretas que conforman un todo $\mathrm{u}$ organizan una entidad individual.

Raven relacionó estos niveles de desarrollo con la edad cronológica como sigue:

Hacia los 3 años, aplicando el test en el formato de tablero, el niño logra acoplar una de las alternativas en el espacio libre del modelo. Al principio, toda pieza que se puede acoplar es gratificante y el niño prueba con diferentes piezas como si se sorprendiera de que todas se 
pueden acoplar en el espacio libre. Algunos niños completan bien los dos o tres primeros elementos de la Serie A poniendo la pieza correcta.

A los 4 años, normalmente el niño se da cuenta de que una de las piezas se parece a la matriz superior. Al principio es suficiente la semejanza (porque no presta atención al tamaño o la orientación de la figura). A menudo el niño coloca una serie de líneas horizontales con una serie similar de líneas verticales y se queda muy satisfecho con lo que ha hecho, incluso después de ver el resultado final. En una fase posterior de desarrollo atiende también al tamaño y orientación de la figura, y el niño es consciente de esos aspectos cuando elige una pieza para completar la matriz. El aprendizaje a partir del resultado de colocar una pieza en la matriz aparece en una fase posterior. A partir de esta fase el "ensayo y error" muestra al niño un modo de trabajo y sólo hay que decirle que, en la medida de lo posible, elija la pieza correcta al primer intento.

A la edad de 5 años, los niños a veces se quedan satisfechos si la alternativa completa la matriz sólo en una dirección. Después, comienzan a buscar la pieza que la completa en las dos direcciones a la vez.

A los 6 años, a menudo los niños seleccionan las figuras que completan la matriz correctamente. Es en esta fase del desarrollo en la que, independientemente de la edad, el niño poco dotado comienza a equivocarse. Se hacen esfuerzos para replicar parte del patrón dado y esto se adopta como un método rutinario de trabajo. Aun cuando la colocación de la pieza da un resultado falso, el niño se siente satisfecho.

A los 7 años de edad, los niños son capaces de concebir objetos concretos (como los de la Serie $\mathrm{Ab}$ ) como un conjunto espacial relacionado, pero aparentemente encuentran dificultad para analizarlo en sus componentes. Si no se elige una pieza que repite una de las tres partes de la matriz, puede serlo aquella otra que reproduce el total de la matriz, como en el caso del $4^{\circ}$ elemento de la Serie $\mathrm{Ab}$ de las matrices a colores, se elige la segunda alternativa. La capacidad para seleccionar aquella parte que permite completar una matriz concebida como un todo surge algo más tarde.

A los 8 años, las dificultades comienzan principalmente hacia el final de la serie 3 del Test de Matrices Progresivas a Colores. Los ejemplos introductorios diseñados para dar entrenamiento en el método de razonamiento no parecen ayudar a los niños a resolver estos problemas. En lugar de seleccionar la figura adecuada, el niño repite una de las figuras dadas en la matriz a completar y adopta esto como método apropiado de trabajo.

Los niños de 7 años y medio y los de 8 años que consideran que se requieren figuras correlativas para completar las matrices, son capaces de resolver muchos de los problemas de la Escala General, de modo parecido a como lo hace un adulto. Podría ser que, hasta que se desarrolla esta capacidad, los elementos situados hacia el final de la serie B de las matrices a colores son muy poco significativos, pero que los mismos elementos son muy evidentes para quienes han desarrollado dicha capacidad. Es muy difícil construir elementos intermedios entre aquellos en los que el éxito depende de seleccionar una figura que complete un grupo de figuras discretas concebidas con un conjunto espacialmente relacionado y aquellos en los que hay que comparar dos figuras e inferir una cuarta a partir de la naturaleza de una tercera. Para una mente madura estos dos tipos de problemas parecen similares y aparentemente se resuelven mediante una línea de razonamiento más o menos igual.

La edad concreta en la que aparece en el niño la capacidad para razonar mediante analogías, es menos importante que la capacidad de pensamiento abstracto que el sujeto mostrará con posterioridad. 
A partir de los 10 años, las respuestas al test de los sujetos que obtienen puntuaciones bajas, medias o altas serán cada vez más diferentes. Cuando los niños son incapaces de resolver un problema tienden a repetir lo que han percibido o asimilado. También tienden a asimilar el campo perceptivo menos adecuadamente, a hacer elecciones arbitrarias según la proximidad entre la figura y el espacio a completar y, menos frecuentemente aún, según su proximidad a ella misma.

El test de matrices progresivas a colores es un instrumento que está dirigido a medir el desarrollo intelectual en niños desde los 5 años y medio hasta los 11 años de edad, o personas con una dotación intelectual baja (Raven et al., 1996; Anastasi y Urbina, 1998).

La presente adaptación se ha llevado a cabo en niños de 6 a 11 años de edad de colegios estatales y no estatales de Lima Metropolitana.

\section{MÉTODO}

\section{Participantes}

El universo de investigación estuvo conformado por los alumnos matriculados en el año 2001 en los diferentes centros educativos de primaria de Lima Metropolitana. En la presente investigación se trabajó con 2496 participantes, el tamaño de muestra se determinó a través del programa informático SOTAM. Para la obtención de la muestra se aplicó un procedimiento de muestreo probabilístico bietápico, en la primera etapa se eligió en cada una de las USES de forma aleatoria los colegios que fueron íncluidos en el estudio y posteriormente en cada colegio se eligió de forma aleatoria los participantes que fueron evaluados.

\section{Tabla $\mathbf{N}^{\circ} \mathbf{1}$}

Composición de la muestra por edad y tipo de colegio

\begin{tabular}{cccc}
\hline \multicolumn{4}{c}{ Tipo de Colegio } \\
\hline Edad & Estatal & No Estatal & Total \\
$\mathbf{6 , 0}-\mathbf{~ 6 , 1 1}$ & 259 & 166 & 425 \\
$\mathbf{7 , 0}-\mathbf{7 , 1 1}$ & 264 & 172 & 436 \\
$\mathbf{8 , 0}-\mathbf{8 , 1 1}$ & 248 & 159 & 407 \\
$\mathbf{9 , 0}-\mathbf{9 , 1 1}$ & 240 & 163 & 403 \\
$\mathbf{1 0 , 0}-\mathbf{~ 1 0 , 1 1}$ & 254 & 163 & 417 \\
$\mathbf{1 1 , 0}-\mathbf{1 1 , 1 1}$ & 245 & 163 & 408 \\
\hline Total & 1510 & 986 & 2496
\end{tabular}

Como se puede observar en todas las edades es mayor el número de participantes de colegios estatales que los de centros educativos no estatales, pues en la población es también mayor la concentración de alumnos que asisten a los colegios estatales.

\section{Instrumento}

\section{El Test de Matrices Progresivas de Raven a Colores}

La versión original de esta prueba fue elaborada por Raven en Gran Bretaña, su forma de aplicación es individual y colectiva, el tiempo de duración es variable entre 40 y 90 minutos. Es una medida de la capacidad de educación de relaciones, uno de los componentes principales de la inteligencia general y del factor "g" (Raven et al., 1996; Anastasi y Urbina, 1998). 
La prueba está conformada por un cuadernillo con tres series de matrices denominadas A, Ab Y B. Cada una de las series tiene 12 elementos, los cuales deben ser contestados en su totalidad. El examinado deberá responder en una hoja de respuestas.

Es un test válido para ser utilizado con niños pequeños y con personas de edad avanzada, también en estudios antropológicos y en la consulta clínica. Puede ser usado satisfactoriamente con gente que, por alguna razón, no pueda comprender o hablar un idioma, sufra algún trastorno físico, sea subnormal o sufra deterioro intelectual. El éxito de la Serie Ab está basado en la capacidad para captar las figuras aisladas como conjuntos especialmente relacionados. Las series A y B cubren todo el proceso cognitivo de que son capaces los niños hasta la edad de 11 años.

Antes que la capacidad para establecer comparaciones y razonar por analogía haya madurado, o en los casos en que se haya deteriorado, las Series A, Ab Y B pueden utilizarse para medir la capacidad que posee una persona para observar y pensar con claridad o para medir el nivel que ha alcanzado su deterioro. Cuando la capacidad para razonar por analogías se ha desarrollado, es más conveniente utilizar la Escala General. Si al aplicar las Series A, Ab Y B se observa que resultan demasiado fáciles, debe aplicarse inmediatamente las Series C, D y E de la Escala General.

La confiabilidad de la prueba original, fue obtenida a través del método de división por mitades obteniéndose índices que varían desde 0,65 hasta un 0,93, mientras que la de testretest, con distintas muestras de niños, varían desde un coeficiente de 0,81 a 0,87 (Ravenetal.,1996).

En cuanto a la validez, los autores de la versión original señalan que los índices de tipo concurrente y predictivo varían con la edad, posiblemente con el sexo, con la homogeneidad de la muestra y con las implicaciones conceptuales de la misma definición de los criterios. En una muestra de 461 casos la prueba alcanzó una validez concurrente con el Goodenough de 0,50. También se ha establecido la validez utilizando el análisis factorial para definir dimensiones superiores de la inteligencia, con saturaciones diferentes según el tipo de muestra de sujetos y de variables introducidas en el análisis (Raven et al., 1996).

\section{RESULTADOS}

\section{Análisis de la confiabilidad}

A continuación se presentan los resultados de los análisis de ítems y los coeficientes de confiabilidad de las partes de la prueba.

En el análisis de ítems de la parte $\mathrm{A}$ (ver tabla $\mathrm{N}^{\circ} 2$ ), se encontró que todas las correlaciones ítem-test corregidas son superiores al criterio propuesto por Kline (1986) de ser mayores a 0.20 , lo cual indica que todos los ítemes son consistentes entre sí.

En el análisis de la confiabilidad del test de matrices progresivas de Raven a colores, permite apreciar que en la parte A se alcanza un coeficiente de Kuder Richardson 20 de 0.72 , lo que permite corroborar la confiabilidad de la primera parte del instrumento. 
Tabla $\mathbf{N}^{\circ} 2$

Análisis de la Con fiabilidad de la parte A del Test de Matrices Progresivas de Raven a Colores

\begin{tabular}{|c|c|c|c|}
\hline Item & Dificultad & .ritc & KR 20 eliminando el ítem \\
\hline $\mathbf{1 A}$ & 0.986 &, $2134 * * *$ & 0.7179 \\
\hline $2 A$ & 0.9864 &, $2136 * * *$ & 0.7151 \\
\hline $\mathbf{3 A}$ & 0.9655 &, $2677 * * *$ & 0.7095 \\
\hline $4 \mathrm{~A}$ & 0.9635 & ,3200*** & 0.7056 \\
\hline $\mathbf{5 A}$ & 0.9435 &, $3615 * * *$ & 0.6998 \\
\hline 6A & 0.8817 &, $4378 * * *$ & 0.6865 \\
\hline 7A & 0.7097 & $0,4918 * * *$ & 0.6742 \\
\hline $8 \mathbf{A}$ & 0.7482 &, $3334 * * *$ & 0.7025 \\
\hline 9A & 0.7666 &, $5045 * * *$ & 0.6723 \\
\hline 10A & 0.7033 &, $5032 * * *$ & 0.6719 \\
\hline 11A & 0.4142 &, $3979 * * *$ & 0.6937 \\
\hline $12 \mathrm{~A}$ & 0.2073 &, $2691 * * *$ & 0.7114 \\
\hline \multicolumn{4}{|c|}{ KR20 = 0,72 } \\
\hline
\end{tabular}

En lo que concierne al análisis de ítems de la parte $\mathrm{Ab}$, en la tabla $\mathrm{N}^{\circ} 3$, se observó que todas las correlaciones ítem-test corregidas también son superiores al criterio de 0.20 , lo cual corrobora que todos los ítems son consistentes entre sí.

Tabla $\mathbf{N}^{\circ} 3$

Análisis de la confiabilidad de la parte Ab del Test de Matrices

Progresivas de Raven a Colores

\begin{tabular}{|c|c|c|c|c|c|c|c|}
\hline Ítem & Dificultad & .ritc & $\begin{array}{l}\text { KR20 } \\
\text { eliminando } \\
\text { el ítem }\end{array}$ & Ítem & Dificultad & .ritc & $\begin{array}{c}\text { KR20 } \\
\text { eliminando } \\
\text { el ítem } \\
\end{array}$ \\
\hline $1 \mathrm{Ab}$ & .9663 & $.3449 * * *$ & .8088 & 1B & .9547 & $.2562 * * *$ & .8203 \\
\hline $2 \mathrm{Ab}$ & .9366 & $.3767 * * *$ & .8056 & $2 B$ & .9146 & $.3602 * * *$ & .8147 \\
\hline $3 \mathrm{Ab}$ & .9463 & $.3392 * * *$ & .8078 & $3 B$ & .8601 & $.4220 * * *$ & .8102 \\
\hline $4 \mathrm{Ab}$ & .8055 & $.5827 * * *$ & .7868 & $4 B$ & .8244 & $.4519 * * *$ & .8077 \\
\hline $\mathrm{SAb}$ & .8123 & $.5626 * * *$ & .7888 & SB & .6596 & $.5272 * * *$ & .8008 \\
\hline $6 \mathrm{Ab}$ & .7498 & $.6138 * * *$ & .7828 & $6 \mathrm{~B}$ & .5345 & $.4309 * * *$ & .8105 \\
\hline $7 \mathrm{Ab}$ & .7574 & $.5934 * * *$ & .7849 & $7 \mathrm{~B}$ & .4888 & $.4436 * * *$ & .8093 \\
\hline $8 \mathrm{Ab}$ & .6175 & $.5647 * * *$ & .7871 & $8 B$ & .3641 & $.5750 * * *$ & .7962 \\
\hline $9 \mathrm{Ab}$ & .5834 & $.5148 * * *$ & .7926 & $9 \mathrm{~B}$ & .3949 & $.6059 * * *$ & .7930 \\
\hline $10 \mathrm{Ab}$ & .6010 & $.4680 * * *$ & .7976 & $10 \mathrm{~B}$ & .4940 & $.5863 * * *$ & .7949 \\
\hline $11 \mathrm{Ab}$ & .5277 & $.4326 * * *$ & .8016 & 11B & .4090 & $.6239 * * *$ & .7911 \\
\hline $12 \mathrm{Ab}$ & .3753 & $.2426 * * *$ & .8203 & $12 \mathrm{~B}$ & .1760 & $.3283 * * *$ & .8168 \\
\hline \multicolumn{4}{|c|}{$\mathrm{KR} 20=0.81$} & \multicolumn{4}{|c|}{$\mathrm{KR} 20=0.82$} \\
\hline \multicolumn{4}{|c|}{$\begin{array}{l}* * * \mathbf{P}<.001 \\
\mathrm{n}=2496\end{array}$} & \multicolumn{4}{|c|}{$\begin{array}{l}* * * \mathbf{P}<.001 \\
\mathbf{n}=\mathbf{2 4 9 6}\end{array}$} \\
\hline
\end{tabular}

Tabla $\mathbf{N}^{\circ} 4$

Análisis de la confiabilidad de la parte B del Test de Matrices

Progresivas de Raven a Colores 
El análisis de la confiabilidad del test de matrices progresivas de Raven a colores, permite apreciar que en la parte Ab se alcanza un coeficiente de Kuder Richardson 20 de 0.81 , lo que permite corroborar la confiabilidad de la segunda parte del instrumento.

En lo que respecta al análisis de ítemes de la parte $\mathrm{B}$ (ver tabla $\mathrm{N}^{\circ} 4$ ), se encontró que todas las correlaciones ítem-test corregidas calculadas son superiores al criterio de 0.20 , lo cual permite concluir que todos los ítemes son consistentes entre sí.

El análisis de la confiabilidad del Test de Matrices Progresivas de Raven a Colores, permite apreciar que en la parte B se obtiene un coeficiente de Kuder Richardson 20 de 0.82 , lo cual permite indicar que la tercera parte del instrumento es confiable.

Finalmente se realizó el análisis de la confiabilidad de toda la prueba, observándose que alcanza un valor de KR20 $=0.91$, el cual puede clasificarse como una alta confiabilidad, por lo que se puede concluir que la prueba de Matrices Progresivas de Raven a Colores permite obtener puntajes confiables.

\section{Análisis de la Validez de Constructo}

Los resultados del análisis de la validez de constructo, efectuado a través del análisis factorial de las áreas de la prueba de matrices progresivas de Raven a colores (ver Tabla $\mathrm{N}^{\circ}$ 5), permitió apreciar que se alcanza una medida de adecuación del Muestreo de KaiserMeyer-Olkin de 0.71 y una test de esfericidad de Bartlett que es significativo, hallazgos que corroboran la pertinencia de la ejecución del análisis factorial. Este análisis se desarrolló a través del método de factorización de los componentes principales, notándose la existencia de un solo factor, el cual permite explicar el $76 \%$ de la varianza de las puntuaciones. Este hallazgo permite concluir que los aspectos evaluados por las tres áreas son similares entre sí y por lo tanto la prueba presenta validez.

Tabla $\mathbf{N}^{\circ} 5$

Análisis de la validez de constructo del test de Matrices Progresivas de Raven a Colores a través del análisis factorial

\begin{tabular}{cccc}
\hline Área & M & D. E & Factor 1 \\
\hline $\mathrm{A}$ & 9.28 & 2.09 & 0.9 \\
$\mathrm{Ab}$ & 8.68 & 2.81 & 0.88 \\
$\mathrm{~B}$ & 7.07 & 2.98 & 0.84 \\
\hline \multicolumn{2}{l}{ Varianza explicada } & $76,32 \%$ & \\
\hline
\end{tabular}

Medida de adecuación del muestreo de Kaiser-Meyer-Olkin= 0,71

Test de esferecidad de Bartlett $=3234,35^{* * *}$

$$
\begin{gathered}
* * * \mathbf{p}<\mathbf{0 , 0 0 1} \\
\mathbf{n}=\mathbf{2 4 9 6}
\end{gathered}
$$

\section{Baremos de la prueba}

En el anexo 1 se incluye la tabla de los rangos percentiles del Test de Matrices Progresivas de Raven a colores, correspondientes para los niños y niñas de 6, 7, 8, 9, 10 Y 11 años de edad de colegios estatales y no estatales de Lima Metropolitana.

Estos rangos percentilares permiten ubicar a los escolares según su capacidad en uno de los siguientes grados de capacidad intelectual (Raven et al., 1996; Ivanovic et al., 2000): 
Grado I Intelectualmente superior. Si el percentil alcanzado es igualo superior a 95.

Grado II Definitivamente superior al término medio. Cuando el percentil obtenido es $\geq 75$ y $<95$.

Grado III Intelectualmente promedio. Si el percentil cae entre los percentiles $>25$ y $<75$.

Grado IV Decididamente inferior al término medio. Si el percentil alcanzado cae entre los percentiles $>5 \mathrm{y} \leq 25$.

Grado V Intelectualmente deficiente. Si el percentil es $\leq 5$.

\section{Análisis Comparativo}

Antes de efectuar el análisis comparativo de acuerdo a las diferentes sociodemográficas seleccionadas, se procedió a verificar la existencia de distribuciones normales en los puntajes de la prueba y las partes que la componen con la finalidad de seleccionar el análisis estadístico más adecuado a la calidad del dato obtenido. Los resultados observados en la Tabla $\mathrm{N}^{\circ} 6$, indicaron que todos valores del estadístico $\mathrm{Z}$ de Kolmogorov Smirnov son significativos $(\mathrm{p}<.001)$ concluyéndose por tanto que los datos no se aproximaban adecuadamente a la distribución normal, por lo que siguiendo a Siegel y Castellan (1995) se aplicaron estadísticos no-paramétricos para realizar los contrastes.

\section{Tabla $\mathbf{N}^{0} 6$}

Análisis de la bondad de ajuste a la curva normal de las partes y el puntaje total de la prueba de Matrices Progresivas de Raven a

Colores

\begin{tabular}{cccc}
\hline Factor & $\mathbf{M}$ & D.E. & KS-Z \\
\hline Parte A & 9.28 & 2.09 & $9,062^{* * *}$ \\
Parte Ab & 8.68 & 2.81 & $8,306 * * *$ \\
Parte B & 7.07 & 2.98 & $5,353^{* * *}$ \\
\hline Total & 25.03 & 6.89 & $4,386 * * *$ \\
\hline$* * * \mathbf{p}<, \mathbf{0 0 1}$ & & & \\
$\mathbf{n = 2 4 9 6}$ & & &
\end{tabular}

\section{Comparación por tipo de colegio}

En la comparación por tipo de colegio (ver tabla $\mathrm{N}^{\circ} 7$ ), se observa que existen diferencias significativas entre los alumnos procedentes de colegios estatales y no estatales tanto en las partes $\mathrm{A}(\mathrm{Z}=-7.09 ; \mathrm{P}<.001), \mathrm{Ab}(\mathrm{Z}=9.87 ; \mathrm{p}<.01)$ y $\mathrm{B}(\mathrm{Z}=-9.27 ; \mathrm{p}<.001)$, así como en el puntaje total $(Z=-10.31 ; P<.001)$. Notándose que en todos los casos el rendimiento es superior en los alumnos procedentes de colegios no estatales. 
Tabla $\mathbf{N}^{\circ} 7$

Prueba U de Mann-Whitney del Tests de Matrices

Progresivas de Raven por tipo de colegio

\begin{tabular}{|c|c|c|c|c|c|}
\hline \multirow{2}{*}{ Varíable } & $\begin{array}{l}\text { Estatal } \\
\mathrm{n}=1509\end{array}$ & $\begin{array}{c}\text { No Estatal } \\
\mathrm{n}=986\end{array}$ & \multirow{2}{*}{$\mathbf{U}$} & \multirow{2}{*}{$\mathbf{W}$} & \multirow{2}{*}{$\mathbf{Z}$} \\
\hline & Media de Rangos & Media de Rangos & & & \\
\hline ParteA & 1167.42 & 1371.32 & 622339.5 & 1761635 & $-7.09 * * *$ \\
\hline ParteAb & 1140.47 & 1422.68 & 571701.0 & 1710996 & $-9.87 * * *$ \\
\hline ParteB & 1127.92 & 1412.56 & 581681.0 & 1720976 & $-9.27 * * *$ \\
\hline Total & 1127.92 & 1431.77 & 562738.5 & 1702034 & $-10.31 * * *$ \\
\hline
\end{tabular}

\section{Comparación por sexo}

Con respecto a la comparación por sexo, en la tabla $\mathrm{N}^{\circ} 8$, se observa que existen diferencias estadísticamente significativas entre los varones y las mujeres en las partes $\mathrm{A}$ $(\mathrm{Z}=2.64 ; \mathrm{p}<.01), \mathrm{Ab}(\mathrm{Z}=3.39 ; \mathrm{p}<.01), \mathrm{B}(\mathrm{Z}=2.38 ; \mathrm{P}<.05)$ y el puntaje total $(\mathrm{Z}=3.03 ; \mathrm{p}$ $<.00)$. Notándose en todos los casos, un rendimiento superior de los varones con respecto a las mujeres.

Tabla $\mathbf{N}^{\circ} 8$

Prueba U de Mann-Whitney del Test de Matrices

Progresivas de Raven por sexo

\begin{tabular}{|c|c|c|c|c|c|}
\hline \multirow{3}{*}{ Variable } & $\begin{array}{c}\begin{array}{c}\text { Varón } \\
\mathrm{n}=1242\end{array} \\
\end{array}$ & $\begin{array}{c}\text { Mujer } \\
\mathrm{n}=1253\end{array}$ & \multirow{3}{*}{$\mathbf{U}$} & \multirow{3}{*}{$\mathbf{W}$} & \multirow{3}{*}{$\mathbf{Z}$} \\
\hline & & & & & \\
\hline & $\begin{array}{l}\text { Media de } \\
\text { Rangos } \\
\end{array}$ & $\begin{array}{c}\text { Media de } \\
\text { Rangos }\end{array}$ & & & \\
\hline Parte A & 1285.66 & 1210.67 & 731336.0 & 1516967 & $2.64 * *$ \\
\hline ParteAb & 1296.66 & 1199.77 & 717682.5 & 1503314 & $3.39 * *$ \\
\hline ParteB & 1282.27 & 1214.03 & 735543.5 & 1521175 & $2.38^{*}$ \\
\hline Total & 1291.78 & 1204.60 & 723736.0 & 1509367 & $3.03 * *$ \\
\hline
\end{tabular}

$* * * \mathbf{P}<.001$

\section{DISCUSIÓN}

La adaptación del test de Raven a colores demuestra que la prueba es válida y confiable para niños de 6 a 11 años de centros educativos estatales y no estatales de Lima Metropolitana, estos resultados son consistentes con lo hallado por Ivanovic, Forno, Durán, Hazbún, Castro, e Ivanovic (2000) en escolares de la Región Metropolitana de Chile, con lo reportado por Blumen (2000) en una muestra de niños talentosos en Lima y lo que se reporta en muestras de escolares en Argentina, Uruguay y España (Raven, 1957).

En la comparación de tipo de colegio se observa que existe una diferencia estadísticamente significativa en el rendimiento en la prueba entre los alumnos de centros educativos estatales y no estatales, siendo superior el rendimiento de los alumnos de centros no estatales, lo cual concuerda con lo reportado por Ivanovic, Forno, e lvanovic (2001) 
en cuanto a la existencia de una asociación directa y significativa entre el nivel de CI y las condiciones socioeconómicas y socioculturales de los escolares chilenos, de esta forma los escolares pertenecientes a los estratos más bajos, presentan un menor desarrollo intelectual, hecho que coincide según Ivanovic, et al. (2001) con los hallazgos de otros investigadores tales como Deutsch, Golden y Birns, Durán, Frey y Pinelli, Ross, Lipper y Auld, Duncan, Brooks-Gunn y Klebanov, Frank. Todos ellos señalan a la inteligencia como uno de los principales instrumentos de adaptación del ser humano, adaptación considerada como una respuesta del hombre a las exigencias del medio ambiente material, social o cultural; por lo tanto, las habilidades intelectuales varían según se enfrenten necesidades primarias de sobrevivencia en los sectores socialmente más deprivados, o las necesidades más sofisticadas de otros medios culturales, tales como los de un mayor avance tecnológico. Los niños social y culturalmente deprivados son por lo general educados en hogares que no favorecen la plena expresión del potencial genético, por lo cual su nivel cultural y la precaria estimulación verbal, cognitiva y ambiental, serían los factores de un desarrollo intelectual más lento.

Con respecto al tema, Denney y Heidrich (1990) demostraron que la aplicación de estrategias de modelamiento es un método efectivo para el entrenamiento en la ejecución de las pruebas de Raven tanto en jóvenes, adultos intermedios y adultos mayores. Estos hallazgos indican que los efectos significativos del entrenamiento pueden obtenerse en una sola sesión, con una duración de algunos minutos, lo cual podría estar explicando la ventaja de los niños que reciben algún tipo de entrenamiento previo en la escuela, esto es, básicamente los alumnos de los centros educativos no estatales.

Asimismo, se observan diferencias estadísticamente significativas en los puntajes alcanzados por varones y mujeres, siendo más elevados los puntajes de los varones, hallazgos que también concuerdan con los de Ivanovic et al. (2001), quienes al hacer la comparación por sexo toman en cuenta que el $8.1 \%$ de los varones presentó un CI de Grado I y el $22.9 \%$ de Grado II, cifras que en el caso de las mujeres descendieron significativamente a $5.3 \%$ y $18.8 \%$ respectivamente; por otra parte, el $23.4 \%$ y $7.2 \%$ de las mujeres, presentaron CI de Grado IV y V, respectivamente, tasas que en los hombres fueron de $19.4 \%$ y $5.8 \%$ ( $<<0.001$ ), resultados señalados como coincidentes con los de Buitrón. Asimismo, reportan a investigadores como Jensen y Reynolds, Lynn y Mulhern, Dai y Lynn quienes utilizando el WISC-R, han verificado que los niños tienen mayor habilidad verbal y visuoespacial y las niñas, mayor habilidad de memoria, siendo similares estos resultado entre niños americanos, escoceses y chinos; no obstante, los niños obtienen puntuaciones más altas que las niñas en la mayoría de los subtests.

La similitud de los resultados contribuye con las hipótesis referidas al CI en relación al sexo, podría tener una base biológica. Por otra parte, parece haber un consenso social, con respecto a la existencia de diferencias en el rendimiento de los niños comparado con el de las niñas; no obstante, la polémica es muy amplia respecto a si el origen de estas diferencias es genético o se debe al trato diferencial proporcionado tanto a niños como a niñas, pues tienen procesos de socialización diferentes. Estos planteamientos fundamentarían de alguna manera los hallazgos de la presente investigación.

Cabe mencionar a Ivanovic et al. (2001) reportando la existencia de estudios de 1990 tales como el de Resnick, Stralka, Carter, Ariel, Bucciarelli, Furlough, Evans, Curran y Ausbon señalando que las niñas registran un mayor desarrollo mental, en comparación con 
los niños. Acton y Schroeder (200 1), estarían corroborando la importancia de la percepción sensorial en el desarrollo de la inteligencia, lo cual tendría que tenerse en cuenta como una variable de explicación de las diferencias entre niños y niñas.

Finalmente, es conveniente señalar la necesidad del análisis confirmatorio del Test de Matrices Progresivas de Raven a colores, a fin de poder analizar los modelos que podrían explicar el funcionamiento de esta prueba. El uso del programa EQS, el LISREL, y especialmente el correspondiente al programa Amos (Arbuckle y Wothke, 2001) permitiría este tipo de análisis.

\section{CONCLUSIONES}

a. La prueba de Matrices Progresivas de Raven a colores presenta confiabilidad por consistencia interna, por el método de Kuder Richardson 20.

b. El Test de Matrices Progresivas de Raven a colores presenta validez de constructo, tal como lo señala el análisis de factores respectivo.

c. El baremo está adaptado para ser utilizado en niños de la ciudad de Lima, Perú.

d. Los alumnos de colegios no estatales presentan puntuaciones más altas en el test de Raven que los alumnos de colegios estatales.

e. Los varones alcanzan en la prueba puntajes más altos que las mujeres.

\section{REFERENCIAS BIBLIOGRÁFICAS}

Acton, G.S., y Schroeder, D.H. (2001). Sensory discrimination as related to general intelligence. Intelligence. 29, 263-271.

Aiken, L (1996). Tests psicológicos y evaluación. México: Prentice Hall Hispanoamerica. American Psychological Association (1998). Manual de estilo de publicaciones. México: Manual Moderno.

Anastasi, A., y Urbina S. (1998). Tests psicológicos y evaluación. México: Prentice Hall Hispanoamericana.

Arbuckle, J., Y Wothke, W. (2001). En: www.utexas.edu/cc/stat/tutorials/amos o en: www.smallwaters.com.

Blumen, S. (2000). Identification of and attention for tire highly able in Lima. Nijmegen.

Denney, N., Heidrich, S. (1990). Training Effects on Raven's Progressive Matrices in Young, Middle-Aged, and Elderly Adults. Psychology and Aging. Vol. 5, № 1, 144-145. Ivanovic, R, Forno, H., Durán, M., Hazbún, J, Castro, C., Ivanovic, D. (2000). Estudio de la capacidad intelectual (Test de Matrices Progresivas de Raven) en escolares chilenos de 5 a 18 años I. Antecedentes Generales, Normas y Recomendaciones. Revista de Psicología General y Aplicada. 53 (1), 5-30.

Ivanovic, D., Forno, H., Ivanovic, R. (2001). Estudio de la capacidad intelectual (Test de Matrices Progresivas de Raven) en escolares chilenos de 5 a 18 años II. Interrelaciones con factores socioeconómicos, socioculturales, familiares, de exposición a medios de comunicación de masas, demográficos y educacionales. Revista de Psicología General y Aplicada. 54 (3), 443-465.

Kline, P. (1986). A handbook of construction, Introduction to psychometric design. London: Methuen.

Raven, J.C. (1957). Test de Matrices Progresivas. Escala especial. Buenos Aires: Paidós.

Raven, J. C., Court, J.H., y Raven, J. (1991). Raven manual General overview. Oxford: 
Information Press Ud.

Raven, J.C., Court, J.H., y Raven, J. (1996). Raven matrices progresivas. Escalas: Color (CPM), General (SPM), Superior (APM). Manual. Madrid: TEA Ediciones S.A.

Siegel, S., Castellan, NJ. (1995). Estadística no paramétrica aplicada a las ciencias de la conducta. México: Editorial Trillas.

Tabla $\mathbf{N}^{\circ} 9$

Rangos percentiles por edad del test de Matrices Progresivas de Raven a Colores

\begin{tabular}{cccccccc}
\hline Percentil & 6años & 7años & 8años & 9años & 10años 11años & Percentil \\
\hline $\mathbf{5}$ & 9 & 12 & 14 & 17 & 17 & 22 & 5 \\
$\mathbf{1 0}$ & 11 & 14 & 16 & 20 & 21 & 25 & 10 \\
$\mathbf{1 5}$ & 12 & 16 & 18 & 21 & 22 & 27 & 15 \\
$\mathbf{2 0}$ & & 17 & 20 & 22 & 24 & & 20 \\
$\mathbf{2 5}$ & 13 & 18 & 21 & 24 & 25 & 28 & 25 \\
$\mathbf{3 0}$ & 14 & 20 & 22 & 25 & 26 & 29 & 30 \\
$\mathbf{3 5}$ & 15 & & 23 & 26 & 27 & 30 & 35 \\
$\mathbf{4 0}$ & 16 & 21 & 24 & & 28 & 31 & 40 \\
$\mathbf{4 5}$ & & 22 & 25 & 27 & & & 45 \\
$\mathbf{5 0}$ & 17 & 23 & 26 & 28 & 29 & 32 & 50 \\
$\mathbf{5 5}$ & 18 & 24 & & & 30 & & 55 \\
$\mathbf{6 0}$ & 19 & & & 29 & 31 & & 60 \\
$\mathbf{6 5}$ & & 25 & 27 & & & 33 & 65 \\
$\mathbf{7 0}$ & 20 & 26 & & 30 & 32 & & 70 \\
$\mathbf{7 5}$ & 22 & 27 & 28 & 31 & & 34 & 75 \\
$\mathbf{8 0}$ & & 28 & 29 & 32 & 33 & & 80 \\
$\mathbf{8 5}$ & 24 & 29 & 30 & & & & 85 \\
$\mathbf{9 0}$ & 25 & 30 & 31 & 33 & 34 & 35 & 90 \\
$\mathbf{9 5}$ & 28 & 32 & 33 & 34 & 35 & & 95 \\
$\mathbf{9 9}$ & 31 & 34 & 34 & 35 & 36 & 36 & 99 \\
\hline $\mathbf{M}$ & 17.65 & 22.55 & 24.55 & 27.01 & 28.21 & 30.64 & $\mathrm{M}$ \\
$\mathbf{D . E}$ & 5.5 & 2.84 & 5.7 & 5.46 & 5.67 & 4034 & $\mathrm{D} . \mathrm{E}$ \\
$\mathbf{n}$ & 425 & 436 & 407 & 403 & 417 & 407 & $\mathrm{n}$ \\
\hline $\mathbf{2 4 9 6}$ & & & & & & &
\end{tabular}

$\mathrm{n}=\mathbf{2 4 9 6}$ 